\title{
Shear Stress in the Microvasculature: Influence of Red Blood Cell Morphology and Endothelial Wall Undulation
}

\author{
Brenna E. Hogan • Zaiyi Shen • Hengdi Zhang • Chaouqi Misbah • Abdul I. \\ Barakat
}

Received: date / Accepted: date

\begin{abstract}
The effect of red blood cells and the undulation of the endothelium on the shear stress in the microvasculature is studied numerically using the lattice Boltzmann-immersed boundary method (LB-IBM). The results demonstrate a significant effect of both the undulation of the endothelium and red blood cells on wall shear stress. Our results also reveal that morphological alterations of red blood cells, as occur in certain pathologies, can significantly affect the values of wall shear stress. The resulting fluctuations in wall shear stress greatly exceed the nominal values, emphasizing the importance of the particulate nature of blood as well as a more realistic description of vessel wall geometry in the study of hemodynamic forces. We find that within the channel widths investigated, which correspond to those found in the microvasculature, the inverse minimum distance normalized to the channel width between the red blood cell and the wall is predictive of the maximum wall shear stress observed in straight channels with a flowing red blood cell. We find that the maximum wall shear stress varies several factors more over a range of capillary numbers (dimen-
\end{abstract}

Brenna E. Hogan · Abdul I. Barakat

Hydrodynamics Laboratory (LadHyX), École Polytechnique

Palaiseau, France

Tel.: +33169335268

E-mail: barakat@ladhyx.polytechnique.fr

Hengdi Zhang · Chaouqi Misbah

Laboratoire Interdisciplinaire de Physique (LiPhy), Université Joseph Fourier

Saint-Martin-d'Hères, France

Zaiyi Shen

Laboratoire Ondes et Matière d'Aquitaine (LOMA), Université de Bordeaux

Talence, France sionless number relating strength of flow to membrane elasticity) and reduced areas (measure of deflation of the red blood cell) than the minimum wall shear stress. We see that waviness reduces variation in minimum and maximum shear stresses among different capillary and reduced areas.

Keywords Hemodynamic Forces - Shear Stress · Endothelium · Red Blood Cells

\section{Introduction}

The fluid shear stress due to viscous blood flow is an important quantity in the study of the circulatory system. In the endothelial cells lining the inner surfaces of blood vessels, shear stress regulates important mechanotransduction events that control vessel development during embryogenesis (Culver and Dickinson 2010; García-Cardeña and Slegtenhorst 2016; Roman and Pekkan 2015), regulates vascular tone (Davies 1995; Hahn and Schwartz 2009), and plays a role in the localization of atherosclerotic lesions (Barakat 1999). Endothelial cells show sensitivity to the details of the shear stress applied upon them. In vitro experiments have shown that temporal changes in applied shear stress patterns have an important influence on endothelial cell phenotypic expression (Uzarski et al. 2013). While the transient and spacevarying nature of shear stress is important to endothelial cell function, a study of blood from a continuum point of view will not suffice to bring out these important time and spatial fluctuations due to RBCs. Thus, studies are necessary in order to determine the shear stresses present in the vessel taking into account the particulate nature of the flowing blood.

Due to the fact that RBCs constitute $40-45 \%$ of the total volume of blood under normal conditions, they 
play an important role in hemodynamics. Consequently the presence of RBCs would be expected to have a significant impact on the shear stress at the endothelial cell surface. Furthermore, pathologies of RBCs can affect their deformability and therefore their morphologies and dynamics in blood flow. To date, few studies have addressed the interplay among RBC morphological changes due to pathology, RBC dynamics, and the stresses experienced at the vascular wall. Two particular cases of pathological RBC morphology have received some attention, including the "schizont stage" of malaria, known to lead to reduced $\mathrm{RBC}$ rigidity and morphological changes whereby the RBC assumes a spherical shape. In this stage, RBCs infected by the Plasmodium falciparum (Pf-RBCs) parasite exhibit enhanced cytoadherence to vascular endothelium and other healthy and infected RBCs, which may lead to capillary occlusions (Dondorp et al. 2004). Fedosov et al. (2011) developed an adhesion model of Pf-RBCs as a function of wall shear stress (WSS) and other parameters, studying how WSS affects the propensity of Pf-RBC to adhere to the vessel wall. Similarly, Barabino et al. (1987) studied in vitro how wall shear rate, calculated from the WSS, mediated adherence to the vessel wall in sickle cells and normal RBCs and found that adherence of sickle cells is enhanced at low shear rates but is comparable to that of normal RBCs at high shear rates. The wall shear rate calculated in the study is calculated considering a homogeneous, Newtonian fluid, and what our study suggests is that the RBCs themselves and the details of their shape and rigidity will induce significant alterations in the WSS, especially in the microvasculature. Other RBC pathologies include spherocytosis and elliptocytosis which are caused by genetic defects in intracellular proteins responsible for the structural connections either between the lipid bilayer membrane and the cytoskeleton or within the cytoskeleton, respectively, and lead to altered cell shape. These genetic defects result in morphologies with reduced surface-to-volume ratios, or higher reduced volumes, through a shear stress-driven mechanism known as vesiculation (Diez-Silva et al. 2010). Even below the hemolytic threshold and independent of genetic abnormalities, shear stress has been shown to induce alterations in RBC properties, most notably a deterioration in cellular deformability (Baskurt 2012). Reduced RBC deformability has also been observed in RBCs stored for blood transfusions (Xu et al. 2018). RBC deformability, in addition to hematocrit and RBC size, has been shown to influence platelet adherence to vessel wall, an early step in thrombus formation. This increase is said to be due to increased platelet transport towards the vessel wall induced by the red blood cells (Aarts et al. 1984).
Platelets thus being marginalized to the layer of fluid closest to the wall will be exposed to shear stress gradients generated at the vessel wall (Kroll et al. 1996). Our results show that variation among extremal wall shear stresses experienced among different reduced areas and RBC rigidities lead to important fluctuations in wall shear stress values.

In wishing to understand how different RBC morphologies may affect the wall shear stress at the endothelial surface, we acknowledge an additional complexity in that the endothelium is not a flat surface. Atomic force microscopy (AFM) has been used to image the endothelial surface topography in vitro (Barbee et al. 1994; Oberleithner et al. 2004). Barbee at al. (1994) reported that unsheared cells had an aspect ratio (length/width) of $1.12 \pm 0.31$ and a height of $3.39 \pm$ $0.70 \mu \mathrm{m}$. After exposure to flow, the cells elongated and shrank in height so that the aspect ratio increased to $2.16 \pm 0.53$ and the height decreased to $1.77 \pm 0.52 \mu \mathrm{m}$. Oberleithner et al. (2004) showed endothelial height profiles of around $3 \mu \mathrm{m}$ for unsheared human umbilical vein endothelial cells (HUVECs). Although these experimental results clearly show the non-flat nature of the endothelium, there have been a limited number of theoretical or numerical studies taking into account the waviness of the endothelial cell geometry. Satcher et al. (1992) modeled the wavy surface as a two-dimensional sinusoidal surface and showed through a linear perturbation analysis that the uneven endothelial surface leads to a nonuniform shear stress distribution at the cellular level. Their results showed that the perturbation shear stress due to the wavy surface could reach values as much as a third greater than the stresses experienced in the flat case, with the peak perturbations occurring at the crests of the wavy surface, as would be expected. Barbee et al. (1995) used their AFM measurements to generate three-dimensional geometries of the wavy endothelial surface that were subsequently used in numerical simulations of the wall shear stress. Similar to the findings of Satcher et al. (1992), their results showed large subcellular gradients of wall shear stress. Furthermore, they demonstrated that flow-induced elongation of the endothelial cells had the effect of significantly reducing these gradients. Pontrelli et al. (2011) investigated the wall shear stress in wavy-walled channels using 2D simulations. Although the effect of the particulate nature of blood on wall shear stress was only slightly touched upon, the authors reported that the mean effect of erythrocytes matches well with continuum hydrodynamics, which logically follows since the simulations involved a pressure-driven flow, thereby imposing the average shear stress along the walls. Interestingly, they indicated that they did find that the instan- 
taneous wall shear stress distribution in the presence of RBCs in the flow could not be recovered by a consideration of blood as a continuous medium alone.

In the present work, we use a Lattice BoltzmannImmersed Boundary Method code where RBCs are simulated as discrete, deformable structures interacting with the flow to investigate the interplay between flow in microvessels, RBC dynamics, and the wall shear stress on both a flat and wavy endothelial surface. There have been numerous previous computational studies which focused on different aspects of normal and pathological RBCs in flow, in addition to those mentioned above, (Ye et al. 2015; Ye et al. 2014; Wu and Feng 2013; Fedosov et al. 2011a; Bagchi 2007); however, the interaction of pathological RBCs and the role of RBC rigidity on vessel shear stress has yet to be widely investigated. We show that RBC morphology has a profound effect on the shear stress at the endothelial surface and that endothelial waviness further amplifies these effects.

\section{Modeling Methodology}

\subsection{Lattice Boltzmann Method (LBM) for Fluid Dynamics}

We performed flow simulations using the Lattice Boltzmann scheme with an immersed boundary method. The Lattice Boltzmann method (LBM) has been shown to recover with good approximation the Navier-Stokes equations, in the limit of small Mach (ratio of the speed of a fluid particle in a medium to the speed of sound in that medium) and Knudsen (ratio of the molecular mean free path to the macroscopic characteristic length scale) numbers (Krüger et al. 2009; Succi 2001). The LBM has been widely used to simulate fluid flows due to its straightforward implementation and its greater amenability to parallel computing compared to direct Navier Stokes simulations. The quantity of interest in LBM is the particle distribution function, $f_{i}(\mathbf{x}, t)$, which represents the probability of finding a fluid particle travelling with speed $\mathbf{c}_{\mathbf{i}}$ at node $\mathbf{x}$ at time $t$. In our simulations, we use the two-dimensional 9 speed lattice (D2Q9) such that $i=0,1,, 8$. The evolution equation of the distribution functions in time and space takes the form:

$$
\begin{array}{r}
f_{i}\left(\mathbf{x}+\mathbf{c}_{\mathbf{i}} \Delta t, t+\Delta t\right)-f_{i}(\mathbf{x}, t)=\Omega_{i}\left(f_{i}(\mathbf{x}, t)\right) \\
+F_{\text {ext }, i}
\end{array}
$$

where $F_{\text {ext }, i}$ is the external forcing term related to the imposed pressure gradient and the membrane force. The left-hand side of Eq. (1) represents free streaming, while the right-hand side represents the effect of collisions and external forces acting on the fluid populations. We use the Bhatnagar-Gross-Krook single relaxation time collision model (BGK SRT):

$\Omega_{i}=\frac{-\left(f_{i}(\mathbf{x}, t)-f_{i}^{e q}\right)}{t_{r}}$

As we can see from Eq. (2), there is a single relaxation time, $t_{r}$, towards a local equilibrium, $f_{i}^{e q}$, which is taken as a second-order expansion of the Maxwell-Boltzmann distribution:

$f_{i}^{e q}=\omega_{i} \rho\left[1+\frac{\mathbf{u} \cdot \mathbf{c}_{\mathbf{i}}}{c_{s}^{2}}+\frac{\left(\mathbf{u} \cdot \mathbf{c}_{\mathbf{i}}\right)^{2}}{2 c_{2}^{4}}-\frac{\mathbf{u} \cdot \mathbf{u}}{c_{s}^{2}}\right]$

where $c_{s}=1 / \sqrt{3}$ is the speed of sound in the lattice, $\omega_{i}$ are weight factors; $\omega_{0}=4 / 9$ for the 0 velocity vector, $\omega_{1-4}=1 / 9$ in the horizontal and vertical directions, and $\omega_{5-8}=1 / 36$ in the diagonal directions, and $\mathbf{c}_{\mathbf{i}}$ are the discretized velocities in each of the 9 lattice directions. The relaxation time, $t_{r}$, is related to the kinematic viscosity of the fluid, $\nu$, such that:

$\nu=\frac{\left(c_{s}^{2}\left(2 t_{r}-1\right)\right)}{2}$

The macroscopic quantities including the local density and local fluid velocity are recovered from $f_{i}$ as:

$\rho(\mathbf{x}, t)=\sum_{i} f_{i}(\mathbf{x}, t)$

$\mathbf{u}(\mathbf{x}, t)=\frac{1}{\rho(\mathbf{x}, t)} \sum_{i} f_{i}(\mathbf{x}, t) \mathbf{c}_{\mathbf{i}}$

The halfway bounce-back boundary condition is often used to implement Dirichlet boundary conditions. While this works well when the wall is aligned with the Cartesian mesh and located halfway between the first solid node and its neighbor fluid node, it is insufficient on a curved boundary as is of interest here for a wavy endothelial cell surface. In order to increase the accuracy on a curved boundary, Bouzidi et al. (2001) proposed a treatment of curved walls which involves the bounce-back scheme and interpolations. The wall shear stress, $\tau$, is then found by:

$\tau=\mu \frac{\partial(\mathbf{u} \cdot \hat{t})}{\partial n}$

where $\mu=\nu \rho$ is the dynamic viscosity of the fluid, $\hat{t}$ is the tangent to the curved surface, $n$ is the normal, and $\mathbf{u}$ is the velocity vector at an off-grid position close to the wall. This interpretation involves only the interpolation of the velocity vector at the off-grid position. 
Since we have modeled the wavy surface as a sine function, the tangent can be found analytically. Although it has been shown that the stress tensor can be calculated at each lattice node (Krüger et al. 2009), applying this equation properly would have led to the interpolation of the 9 distribution functions, so instead, we chose to use the macroscopic equation given by Eq. (7) implemented with a finite-difference scheme.

\subsection{RBC Model}

We use a 2D vesicle model. Vesicles consisting of a phospholipid bilayer enclosing an internal fluid share the inextensibility and incompressibility of RBCs; however, they are fluid and lack shear elasticity. Vesicles have been shown to exhibit many similar dynamics and morphologies to red blood cells including equilibrium shapes, slipperlike shapes, and lateral migration; and thus have been used extensively in literature as a model for red blood cells (Vlahovska et al. 2009; Vlahovska et al. 2013). The model of the RBC in our simulations is taken from the $2 \mathrm{D}$ spring model developed by Tsubota et al. (Tsubota and Wada 2010; Tsubota et al. 2006). In order to accurately model the inextensible bilipid membrane of RBCs, constant perimeter is imposed and incompressibility of the enclosed fluid is assured by imposing a constant enclosed area. The coupling of the fluid flow and RBC dynamics is achieved using the immersed boundary method whereby membrane forces are distributed as point forces to nearby fluid nodes and fluid forces are distributed to Lagrangian membrane nodes using a discrete delta function (Kaoui et al. 2011; Peskin 2002; Zhang et al. 2007). The validation of our code can be found in Shen at al. (2017). The problem is characterized by three dimensionless parameters. The first dimensionless quantity is the capillary number defined as:

$C a=\frac{\mu R_{0}^{3} \dot{\gamma}}{B}$

where $R_{0}$ is the effective radius of the RBC and is given by $R_{0}=\sqrt{A_{R B C} / \pi}$ where $A_{R B C}$ is the enclosed area of the RBC, $\dot{\gamma}$ is the shear rate found by $\dot{\gamma}=R_{0} U /(W / 2)^{2}$, where $U$ is the maximum speed in the vessel and $W$ is the width of the channel, and $B$ is the bending modulus of the RBC, the details of which can be found in (Shen et al. 2017). A $C a<1$ indicates that flow conditions are weaker than the bending resistance of the cell, thus an effectively rigid cell, whereas $C a>1$ indicates that flow conditions are stronger than the bending resistance of the cell, thus an effectively soft cell. The second dimensionless parameter is the degree of confinement given by:

$C_{n}=\frac{2 R_{0}}{W}$

The final dimensionless quantity is the reduced area given by:

$\alpha=\frac{A_{R B C}}{A_{c}}=\frac{4 \pi A_{R B C}}{P^{2}}$

which is the ratio of the area enclosed by the RBC, $A_{R B C}$, to that of a circle of area $A_{c}$, having the same perimeter, $P$, as the RBC. A reduced area of 1 corresponds to a circle. The different equilibrium shapes of RBCs under no flow conditions for various reduced areas have been previously reported using the LBM method (Kaoui et al. 2011).

\subsection{Simulation Details}

Simulations were carried out with a D2Q9 lattice grid with periodic boundary conditions in the direction of flow induced by imposing a pressure gradient across the inlet and outlet of the channel and with the Dirichlet no-slip boundary condition at the walls. The microvessel is first represented by a rigid, flat-walled channel and later on by wavy walls given by sinusoidal functions such that the positions of the bottom and top walls are given, respectively, by:

$$
\begin{array}{r}
y_{\text {wall }}^{b}=\frac{A}{2} \sin \left(\frac{2 \pi x}{\lambda}\right)+\frac{A}{2} \\
y_{\text {wall }}^{t}=W-\left(\frac{A}{2} \sin \left(\frac{2 \pi x}{\lambda}\right)+\frac{A}{2}\right)
\end{array}
$$

where $A$ is the amplitude of the undulation and $\lambda$ is the wavelength. The RBC is modeled in $2 \mathrm{D}$ as an elastic membrane filled with a viscous fluid as described above. In our simulations, the fluids separated by the membrane were taken to have the same density and viscosity. In the absence of flow, with a reduced area of 0.7 , the RBC assumes an equilibrium biconcave shape with a length along the major axis which is taken as $\sim 8 \mu \mathrm{m}$ and a length along the minor axis of $\sim 3 \mu \mathrm{m}$. In LBM units, $R_{0}=3 \mu \mathrm{m}$ is represented by 15 discrete meshes in consideration of both efficiency and accuracy. During all simulations in this study, the Reynolds number was held constant such that $R e=U R_{0} / \nu=0.1$ to approximate the Stokes flow in microcirculation. The channel length is chosen as $L=50 R_{0}$ in our simulations. We have validated that this length is long enough to eliminate artifacts due to periodic boundary conditions. 


\section{Results}

3.1 Single RBC in Flat-Walled Channel

We begin by considering an individual RBC flowing through a $12 \mu \mathrm{m}$-wide flat-walled channel and explore the impact of the RBC on the wall shear stress. It has been shown that in terms of the morphology and dynamics of a single RBC in Poiseuille flow, rich dynamics can be seen (Tahiri et al. 2013). As has been previously reported, the morphology of RBCs in confined Poiseuille flow is a function of the three dimensionless parameters $C a, C_{n}$, and $\alpha$ defined above. Among the stable RBC morphologies, several are permanent including the parachute. Therefore, we begin by analyzing the effect on the flow of a single RBC with this morphology. (a)

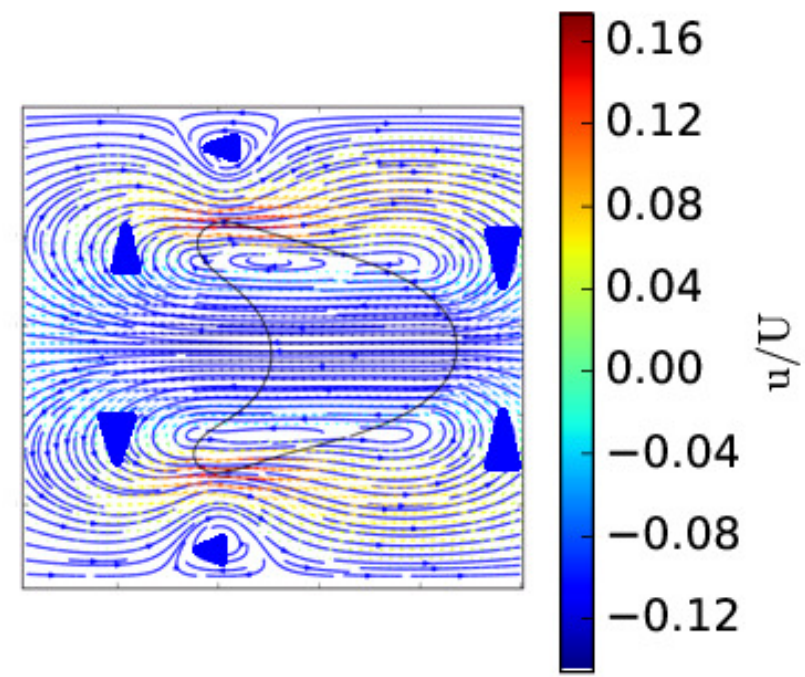

(b)

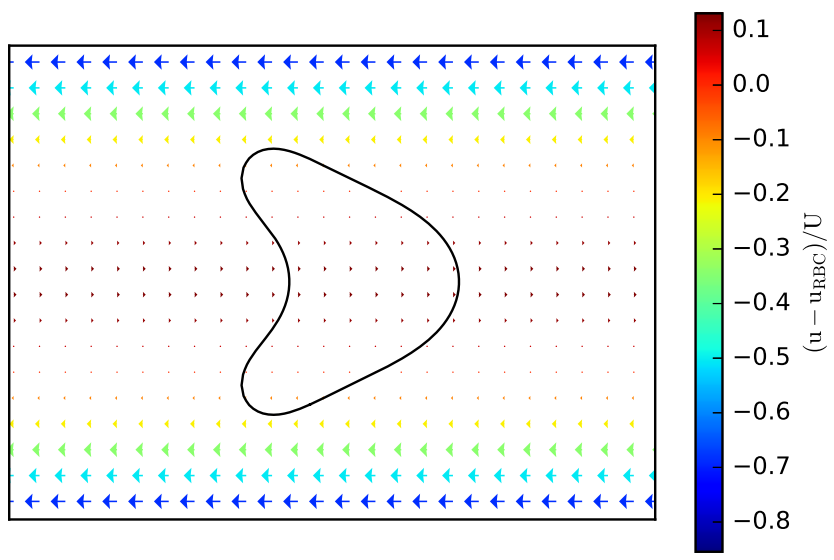

Fig. 1: (a) Streamlines and velocity flow field corresponding to induced flow by a single RBC with a parachute morphology $\left(C_{n}=0.45, C a=10, \alpha=0.7\right)$. Colorbar corresponds to relative magnitude of streamwise velocity to maximal velocity, $U$, in the channel (b) Velocity vectors in the co-moving frame with the $\mathrm{RBC}$ showing zero velocity inside the RBC. Colorbar corresponds to relative magnitude of streamwise velocity in the co-moving frame to maximal velocity.

Figure 1a demonstrates the perturbation or induced flow due to the presence of the parachute-shaped RBC. In our simulations, the shapes of the RBCs are the result of an interplay between the bending and tension forces of the RBC and hydrodynamic forces. For a symmetric parachute shape, there is no tank-treading or normal displacement. Thus, when we plot the velocity in the co-moving frame with the RBC in Fig 1b, we see zero flow field inside, like a rigid particle having the same shape. We investigate the sign of the induced flow for information about how the flow is perturbed due to 
the presence of the RBC. The total flow (TF) is the sum of the imposed flow ( $\mathrm{ImF}$ ) and the induced flow, or the perturbation flow $(\mathrm{PF})$ arising from the elastic forces of the RBC membrane, such that

$T F=I m F+P F$

The sign of PF (positive in the direction of the $\operatorname{ImF}$ which is left to right; negative if against the $\operatorname{ImF}$ ) indicates how the flow is being perturbed by the presence of the RBC. We see in Fig. 1a that the PF generally acts to accelerate the flow near the wall, except at two recirculation zones where the perturbation has resulted in local reversal of the induced flow at the wall. These two zones correspond to the point where the cell is closest to the wall, an observation which was also previously reported in (Freund and Vermot 2014; Yin and Zhang 2012; Xiong and Zhang 2010). These zones of flow reversal are not observed in the unbounded geometry (Ghigliotti et al. 2012).

Previous work has reported how changes in the degree of confinement (value of $C_{n}$ ) in Poiseuille flow alter RBC morphology (Tahiri et al. 2013). We varied $C_{n}$ in the range of $0.2-0.7$ and examined the effect of three stable RBC morphologies, namely the unconfined slipper, the parachute, and the confined slipper, on the wall shear stress. Figure 2 shows how the RBC morphology affects the wall shear stress footprint normalized to the Poiseuille solution of flow through parallel plates, such that:

$\frac{\tau}{\tau_{\text {Pois }}}=\frac{\tau}{(d P / d x)_{i m} W / 2}$

where $\tau$ is the wall shear stress measured along the wall obtained from simulations, $\tau_{\text {Pois }}$ is the wall shear stress obtained from the Poiseuille solution of flow through parallel plates, and $(d P / d x)_{i m}$ is the imposed pressure drop along the channel axis per unit length. (a)

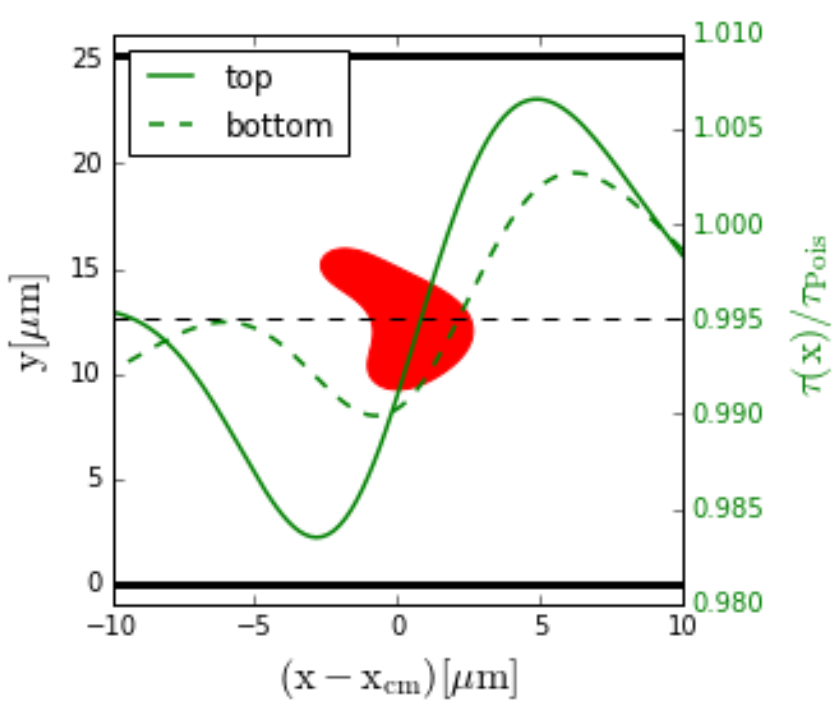

(b)

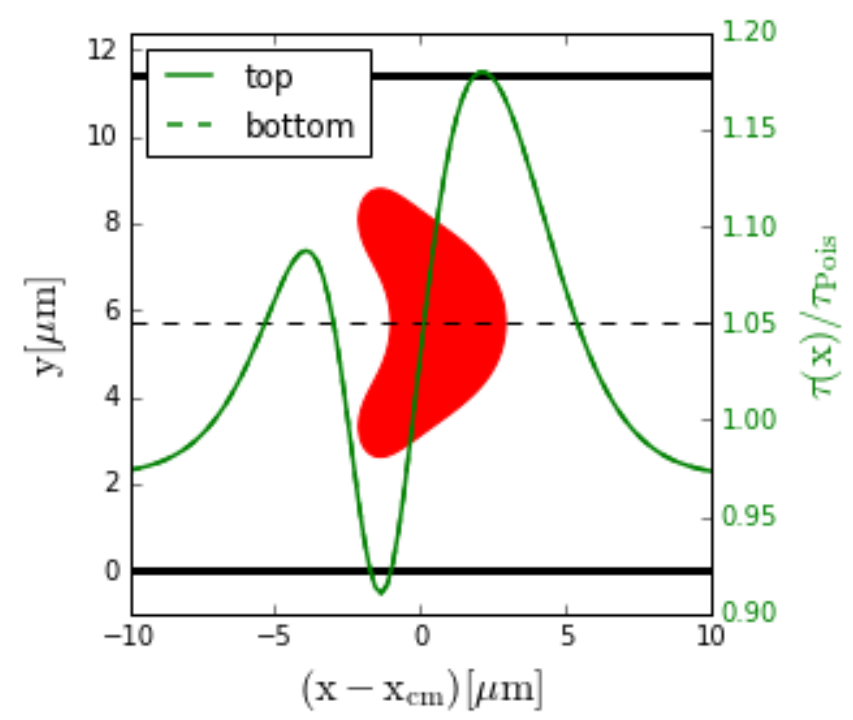


(c)

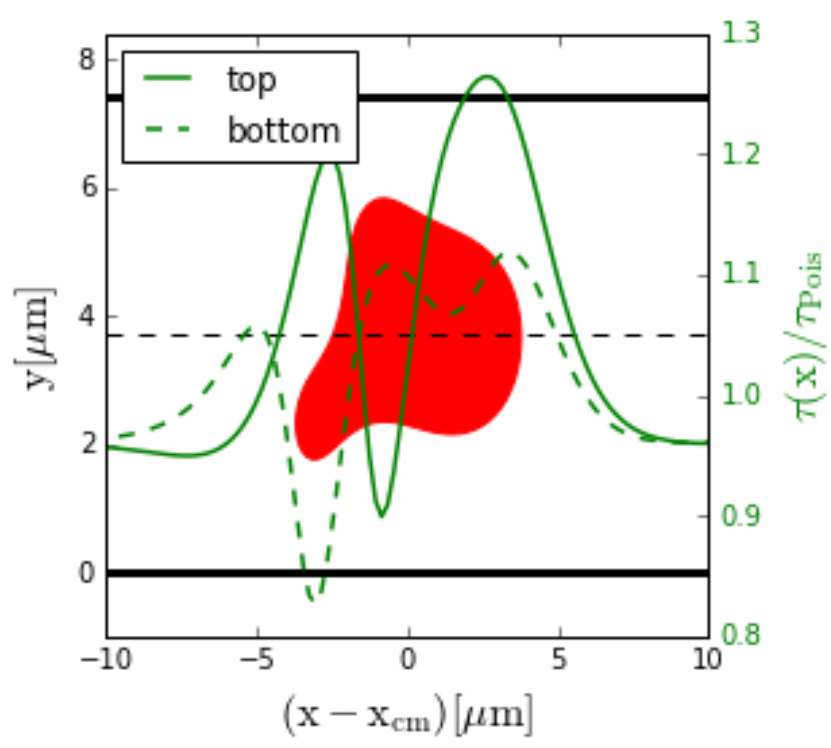

Fig. 2: Effect of RBC morphology on the wall shear stress footprint along the top and bottom walls of the channel. (a) Large channel (small confinement) with RBC exhibiting the unconfined slipper morphology $\left(C_{n}=0.2, \mathrm{CaW} / R_{0}=15\right)$; (b) Intermediate confinement with RBC exhibiting the parachute morphology. Since the parachute is an axisymmetric shape, the wall shear stress curves for the top and bottom walls lie on top of each other $\left(C_{n}=0.45, C a W / R_{0}=15\right)$; (c) Small channel (large confinement) with RBC exhibiting the confined slipper morphology $\left(C_{n}=0.7, \mathrm{CaW} / R_{0}=\right.$ $15)$. Note the different scales for shear stress for optimal visibility of the effect. $x_{c m}$ denotes the x-position of the center of mass of the RBC.

Figure 2 demonstrates that a "peak-valley-peak" form of the wall shear stress generally emerges. This form of the wall shear stress was previously observed in (Yin and Zhang 2012; Xiong and Zhang 2010). Yin et al. (2012) perform a lubrication analysis for a cylinder moving close to a wall in order to investigate the wall shear stress. In obtaining an expression for the pressure along the wall, they find that it is the reverse pressure gradient generated between the moving cell and wall which results in the curious waveform observed in the wall shear stress. In light of their analysis, we extracted the pressure gradient along the wall for each of the three morphologies investigated. (a)

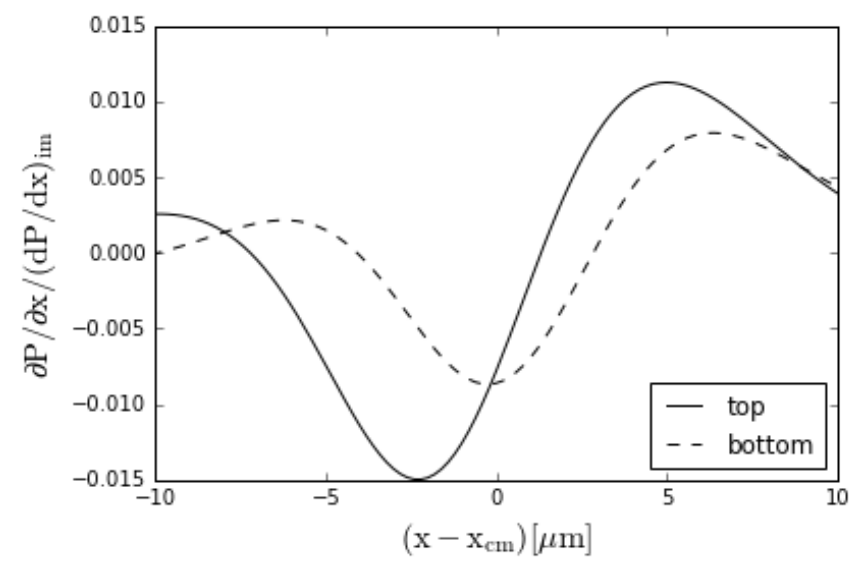

(b)

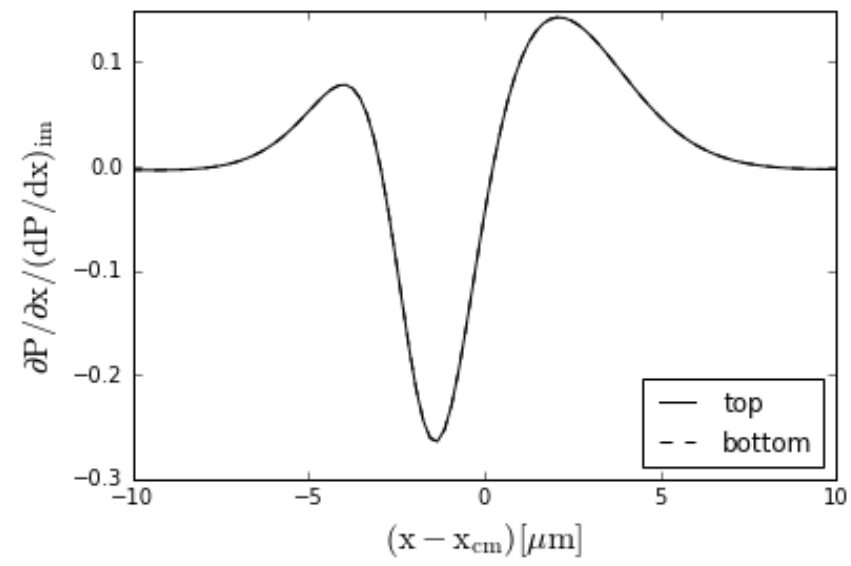

(c)

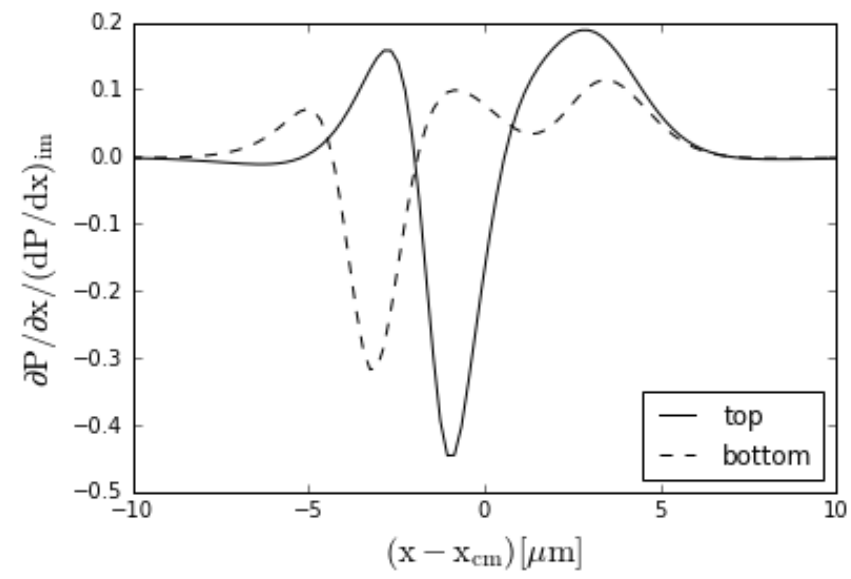

Fig. 3: Pressure gradient along the top and bottom walls of the channel. (a) Large channel (small confinement) with RBC exhibiting the unconfined slipper morphology $\left(C_{n}=0.2, C a W / R_{0}=15\right)$; (b) Intermediate confinement with RBC exhibiting the parachute morphology. Since the parachute is an axisymmetric shape, the curves for the top and bottom walls lie on top of each other $\left(C_{n}=0.45, C a W / R_{0}=15\right) ;(\mathrm{c})$ Small channel (large confinement) with RBC exhibiting the confined slipper morphology $\left(C_{n}=0.7, C a W / R_{0}=15\right)$. 
In Fig 3, we see the features of the WSS are reflected in the plots of the pressure gradient along the wall. Similarly to Yin et al. (2012), we observe the largest pressure gradient opposite to the $\mathrm{RBC}$ motion at the narrowest part between the $\mathrm{RBC}$ and the wall. This reverse pressure gradient generated between the $\mathrm{RBC}$ and the wall impairs the flow velocity and the WSS, consistent with the flow reversal at the narrowest gap observed in Fig 1a of the induced flow. We find the behavior of the WSS captured in the profile of the pressure gradient at the wall for the different morphologies. We see that the pressure gradient and WSS variations are more profound as the size of the channel is decreased, also in accord with observations of Yin et al. (2012).

A summary of the characteristics of the wall shear stress variation is given in Table 1 for the different results presented in Fig. 2, where $\min \left(\tau / \tau_{\text {Pois }}\right)$ corresponds to the minimum wall shear stress observed along either channel wall, while $\max \left(\tau / \tau_{\text {Pois }}\right)$ corresponds to the maximum wall shear stress observed in the channel, IR represents the influencing range, or the width over which the WSS perturbation to the Poiseuille value persists, such that $\left|\left(\tau(x)-\tau_{\text {Pois }}\right) / \tau_{\text {Pois }}\right| \geq 0.02$ from the trailing and leading edges of the cell, corresponding to a minimum of $2 \%$ perturbation from the nominal shear stress, and $\tilde{T}$ is the time duration of the perturbation for a fixed point on the endothelium calculated by $\tilde{T}=I R / V_{R B C}$ where $V_{R B C}$ is the velocity of the RBC. We see that channel size has an inverse effect on the amplitude of WSS perturbation. For the largest channel (case a), we see the smallest variation $0.98-1.01$, while for the smallest channel (case c), we see the largest variation $0.83-1.26$. Considering that the Poiseuille solution for shear stress in the small channel is higher than that in a wider channel with the same mean flow velocity, the absolute variation of the shear stress in the smaller channel is even more significant. The influencing range IR cannot be defined in the case of the unconfined slipper, case a, because the perturbation is always below the threshold. It is largest for the parachute morphology, since we see that its range extends beyond the length of the cell (the second peak is found well after the trailing edge of the cell in Fig. $2 \mathrm{~b})$ ). The influencing range of the confined slipper is different for the top and bottom walls, since the morphology is not axisymmetric. Lastly, the RBCs travel at roughly the same velocity so that the time duration results largely reflect the differences in influencing range.

In order to understand the effect of the morphology of RBCs on the shear stress at the wall, we varied the reduced area, which represents the degree of swollenness of the cell. By varying the reduced area, we can simulate the morphologies of different potential pathological conditions of RBCs. A relatively low confinement vessel, $C_{n}=0.42$, representing a vessel with a width of $12 \mu \mathrm{m}$ is investigated first. As illustrated in Fig. 4a, the $\mathrm{RBC}$ shapes obtained are generally asymmetric.

(a)

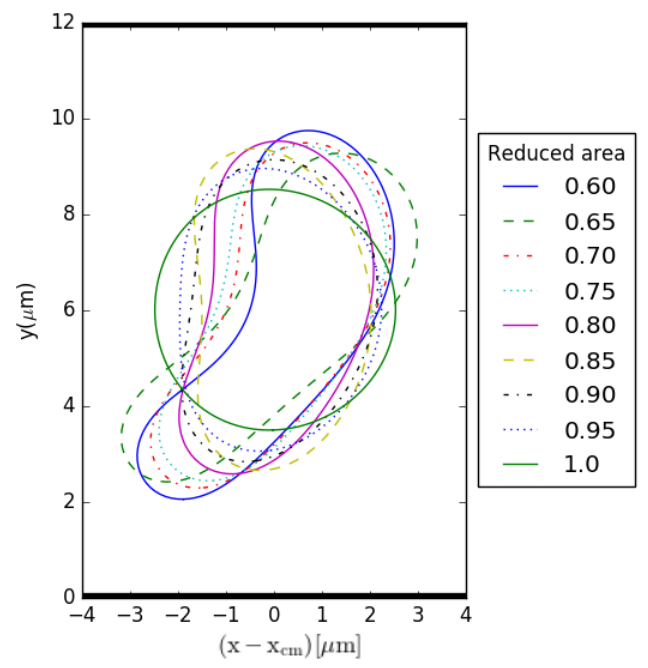

(b)

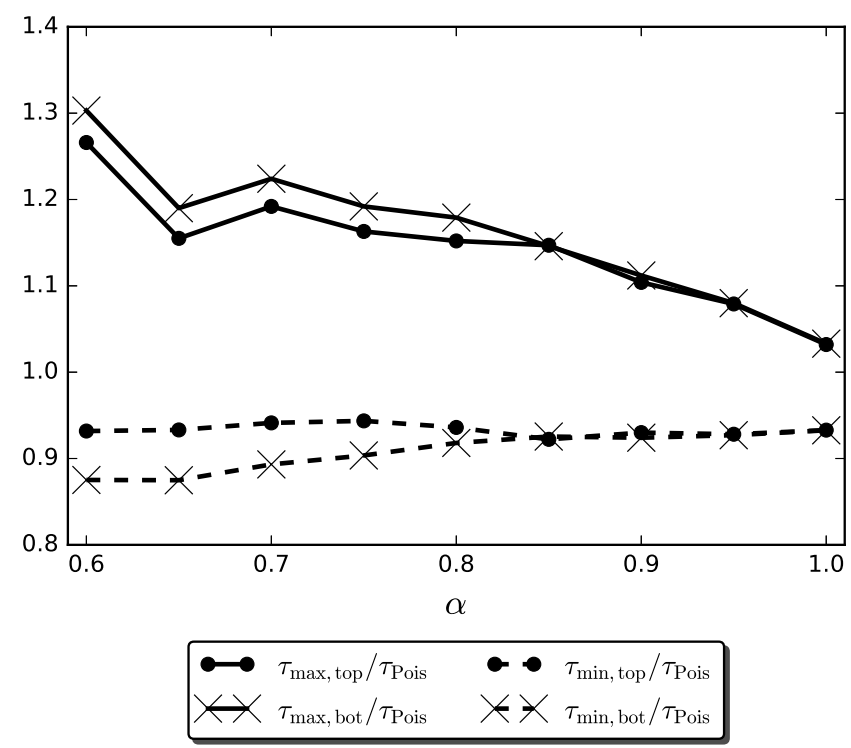


Table 1: Minimum and maximum wall shear stress in the channel, influencing range R, and time duration $\tilde{T}$ for the three different cases represented in Fig. 2. Lowercase and italicized $t$ and $b$ represent top and bottom wall, respectively.

\begin{tabular}{lllll}
\hline Case & $\min \left(\tau / \tau_{\text {Pois }}\right)$ & $\max \left(\tau / \tau_{\text {Pois }}\right)$ & $I R[\mu m]$ & $\tilde{T}[m s]$ \\
\hline $\mathrm{a}$ & $0.98(t)$ & $1.01(t)$ & - & - \\
$\mathrm{b}$ & 0.91 & 1.18 & 15 & $5.80 \mathrm{e}-2$ \\
$\mathrm{c}$ & $0.83(b)$ & $1.26(t)$ & $12.2(t) ; 14.2(b)$ & $4.70 \mathrm{e}-2(t) ; 5.50 \mathrm{e}-2(b)$ \\
\hline
\end{tabular}

(c)

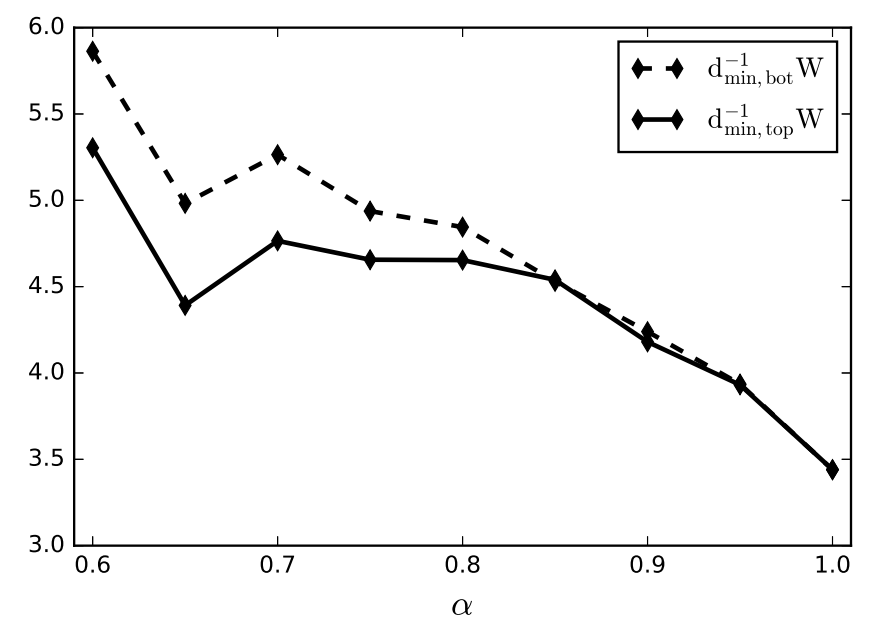

Fig. 4: Effect of reduced area on RBC morphology and wall shear stress for $C_{n}=0.42(W=12 \mu m)$ and $C a=0.1$. (a) Stationary shapes obtained for different values of reduced area; (b) Maximum, $\tau_{\max }$, and minimum, $\tau_{\text {min }}$, shear stress experienced in the channel with a single RBC normalized by the Poiseuille solution. The values for both the top and bottom of the channel are shown; (c) Plot of the normalized inverse minimum distance $d_{\min }^{-1} W$ of the cell to the respective closest wall

Figure 4b suggests that the extremal wall shear stress does not have a clear relationship to reduced area at first glance. We also observe that the symmetry of the cell with respect to the centerline determines whether the values of the shear stress on the top and bottom walls are equal or not. While the maximum shear stress values can range from several to $\sim 30 \%$ greater than the Poiseuille solution in the absence of the RBC; the minimum wall shear stress values vary less than $\sim 11 \%$ over the same range of reduced areas. We hypothesized that the dependence of the maximum wall shear stress on reduced area is determined by the extent of the cell in the channel in the perpendicular streamwise direction. To test this hypothesis, we determined the distance between the closest point of the cell and the channel wall. In Fig. 4c, we plot a quantity related to the instantaneous cell-free layer, or the RBC-free layer of plasma next to vessel walls, such as that previously reported in (Oulaid and Zhang 2015). We calculate from the instan- taneous cell-free layer, the minimum distance between the RBC membrane and the wall, and plot the inverse of this normalized quantity in Fig. 4c. We observe that the inverse normalized minimum distance exhibits exactly the same dependence on reduced area as the maximum wall shear stress (cf: Fig. 4b).

To investigate how robust the results shown in Fig. 4 are, we increased the value of Ca by a factor of 100 (from 0.1 to 10 ), effectively creating a softer cell and repeated the simulations for the same confinement. 
(a)

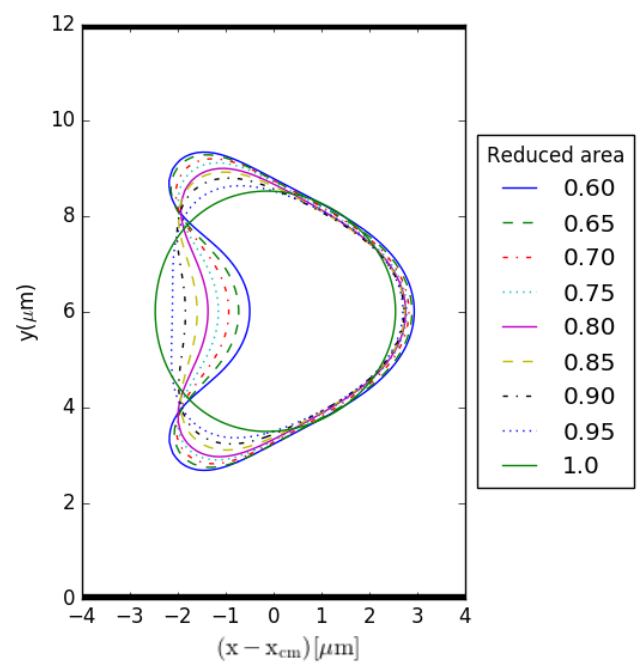

(b)

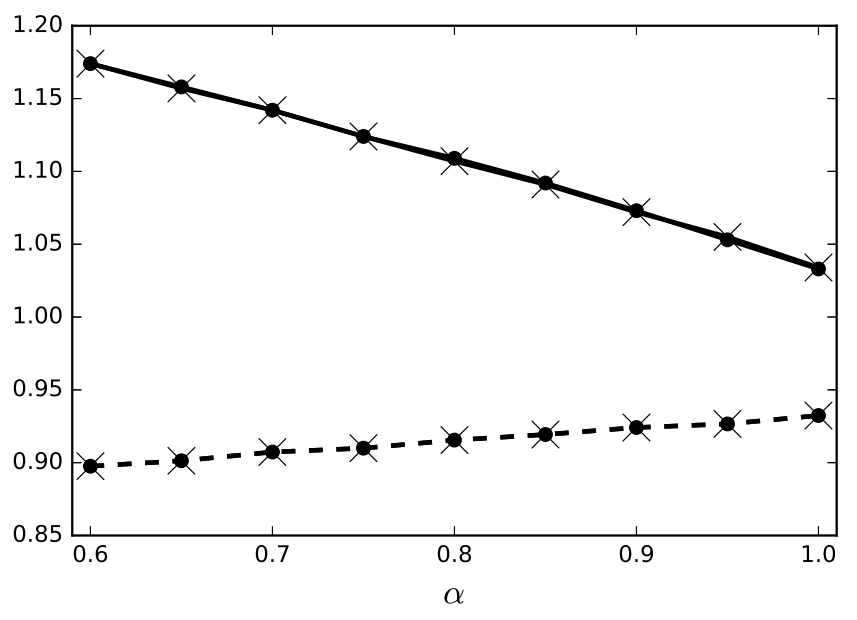

$\longrightarrow \tau_{\text {max top }} / \tau_{\text {Pois }} \bullet \bullet \tau_{\text {min, top }} / \tau_{\text {Pois }}$ (c)

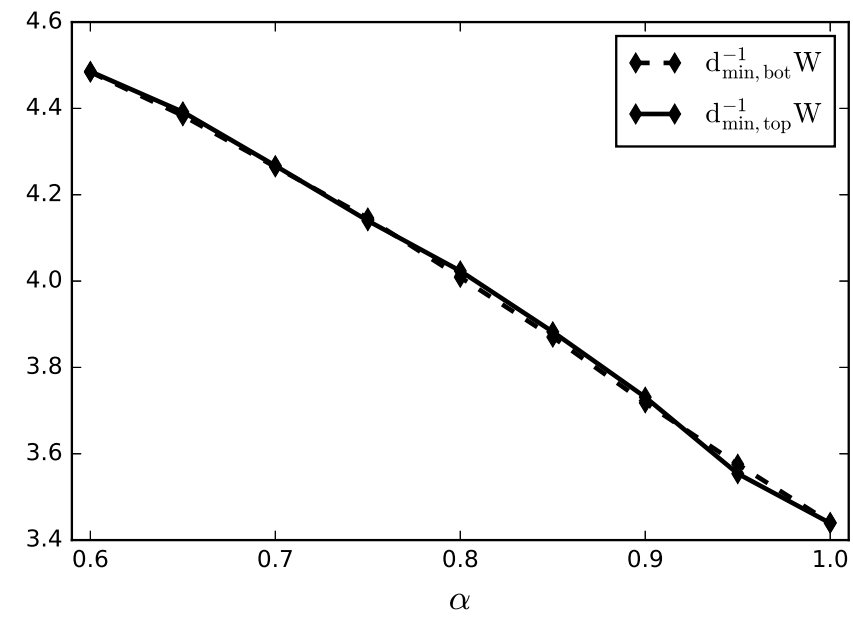

Fig. 5: Effect of reduced area on RBC morphology and wall shear stress for $C_{n}=0.42(W=12 \mu m)$ and $C a=10$. (a) Stationary shapes obtained for different values of reduced area; (b) Maximum and minimum shear stress experienced in the channel with a single RBC normalized by the Poiseuille solution. The values for both the top and bottom of the channel are shown; (c) Plot of the inverse minimum distance $d_{\min }^{-1} W$ of the cell to the respective closest wall.

Figure 5a demonstrates that the RBC morphologies for all reduced areas are axisymmetric, thus leading to equal values of the extremal shear stresses and inverse distance along the top and bottom walls. As illustrated in Fig. 5b and c, the dependence of the maximum wall shear stress on reduced area matches exactly that of the inverse of the minimum distance. Thus, this minimum distance is the physical parameter that drives the maximum shear stress regardless of RBC rigidity. We see that the point closest to the wall is further from the wall for larger reduced areas, shown in Fig. 5c. We again observe a larger variation in maximum wall shear stress, $\sim 17 \%$, while the minimum wall shear stress varies only at most $\sim 10 \%$ from the no-cell Poiseuille solution.

Comparing Figs. 4b and 5b, we observe that for the same confinement, the wall shear stress is lower for a softer RBC (except for $\alpha \approx 1$ in which case the morphologies are the same and therefore the distance to the wall equal). Freund et al. (2014) also observe that rigid cells induce greater perturbations to the wall shear stress. In our analysis, we show that the morphologies adopted for a larger capillary number have minimum distances that are further from the wall than those observed for the morphologies adopted in the case of a smaller capillary number. The cells become more elongated in the perpendicular flow-wise direction in the case of the smaller capillary number and therefore the distances between the cell and the wall are smaller. 
Finally, we examine the effect of confinement by considering a smaller vessel of $7 \mu \mathrm{m}$ in width. (a)

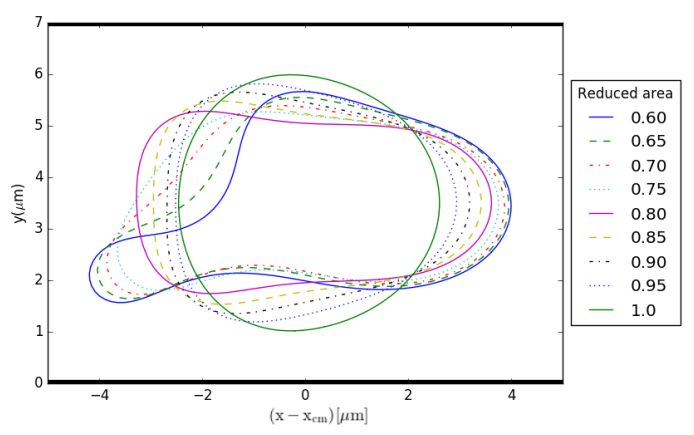

(b)

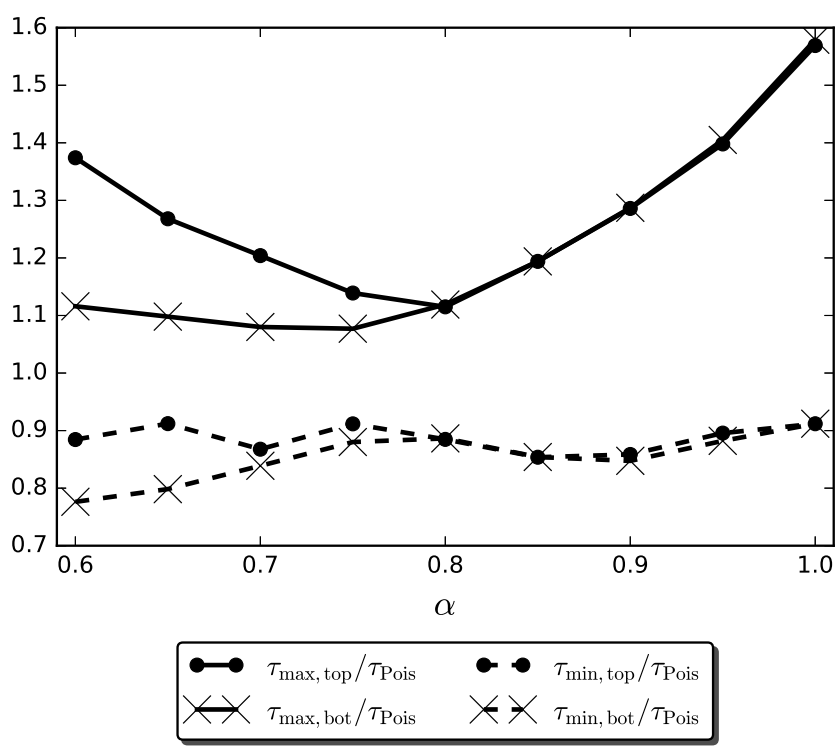

(c)

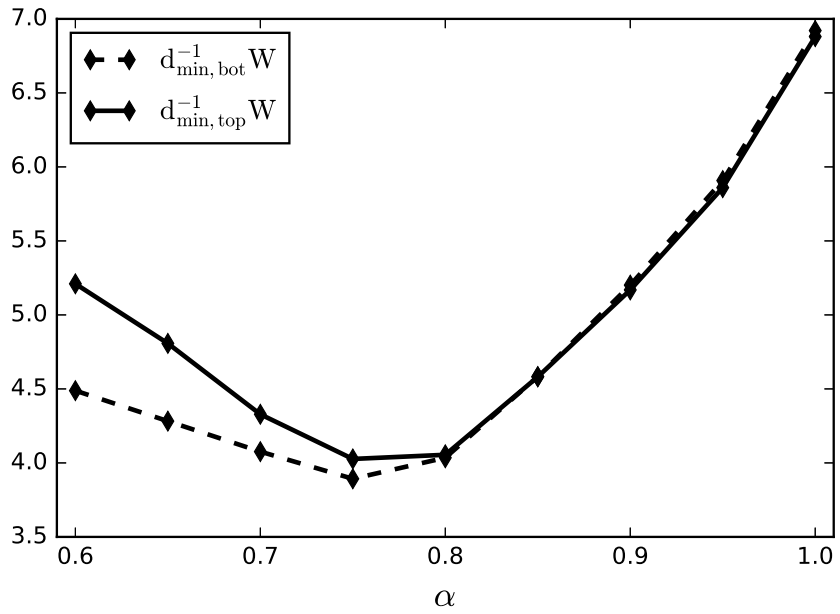

Fig. 6: Effect of reduced area on RBC morphology and wall shear stress for $C_{n}=0.72(W=7 \mu m)$ and $C a=$ 10. (a) Stationary shapes obtained for different values of reduced area; (b) Maximum and minimum shear stress experienced in channel with one RBC normalized by the Poiseuille solution. The values for both the top and bottom of the channel are shown; (c) Plot of the inverse of the normalized minimum distance, $d_{\min }^{-1} W$, of the cell to the respective closest wall. 
Figure 6a demonstrates that an asymmetric slipper shape is observed for $\alpha<0.80$. At $\alpha=0.80$, the slipper shape becomes axisymmetric, and for all reduced areas beyond this value, symmetric morphologies are obtained. The difference in RBC morphology behavior above and below $\alpha=0.80$ leads to the branches found in the plots of the maximum and minimum wall shear stresses observed in Fig. 6b, where the maximum shear stress along the top wall is identical to that along the bottom wall for $\alpha>0.80$ but not so for reduced areas below this cutoff. Again, the maximum shear stress shows more variation, greater than $50 \%$, while the minimum wall shear stress varies at most $\sim 21 \%$ for the same range of reduced areas. Finally, Fig. 6c demonstrates that here again, $\left(d_{\min } / W\right)^{-1}$ is the physical determinant of the wall shear stress behavior observed in Fig. 6b. Comparing Fig. 6b to Figs. 4b and 5b, we see that the maximum shear stresses are higher in a smaller vessel than in a larger vessel. We also see again that RBCs are closer to the wall in the case of the smaller channel. Interestingly, we see that among the reduced areas, that corresponds the most to a circle exhibits the smallest maximal wall shear stress fluctuation in the case of the $12 \mu \mathrm{m}$ channel; however, in the case of the smaller $7 \mu m$ channel, it exhibits a much larger wall shear stress than all the other reduced areas due to its greater extension into the channel than the other RBCs which instead elongate in the streamwise direction. This behavior could be of interest in the study of the schizont stage in malaria.

We recast the results in Figs. 4, 5, and 6 onto a single graph plotting $\tau_{\max } / \tau_{\text {Pois }}$ vs $\left(d_{\min } / W\right)^{-1}$.

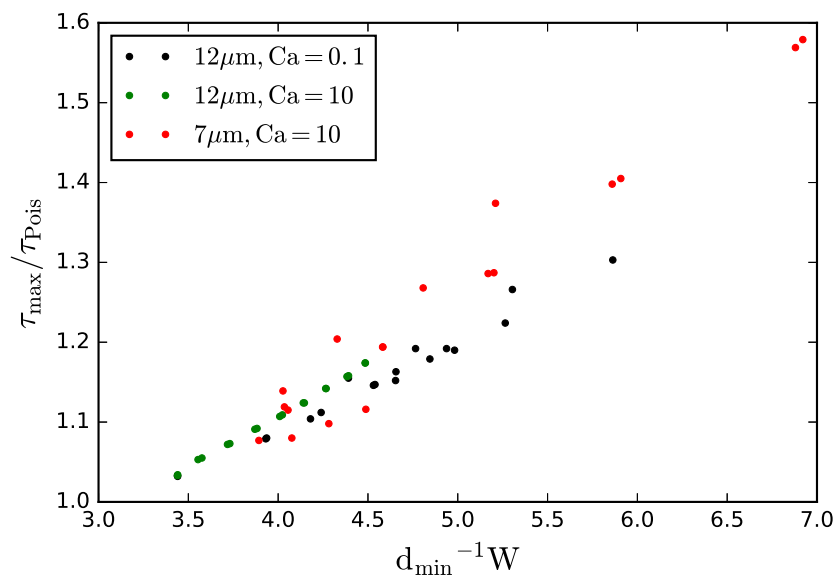

Fig. 7: Recasting results from Figs. 4c, 5c, and 6c such

\section{that $\tau_{\max } / \tau_{\text {Pois }}$ vs $d_{\min }^{-1} W$.}

The inverse minimum distance normalized to the width of channel appears to be an accurate predictor of the normalized maximal wall shear stress in the channel, irregardless of cell rigidity and channel size. We note one possible cause of variation from a strictly linear relationship could be from the different resistance produced by different morphologies of RBCs which would result in different nominal shear stresses in the channel for a given imposed pressure gradient for which our model does not take into account.

\subsection{Effect of a Wavy Wall on Wall Shear Stress}

At the scale of the microcirculation, the endothelium's wavy topography becomes important, and a pertinent question then becomes what effect the vessel waviness has on the flow field. Sinusoidal curves are implemented as boundaries. The magnitude of shear stress can be extracted from simulations using bilinear interpolation of velocities located on grid points close to the wall, as explained in the Modeling Methodology section. For the time being, we will keep the sine curves on the top and bottom boundaries in phase. The effect of dephasing will be studied later. We simulate a channel with wavy walls, with effective width of $12 \mu \mathrm{m}$ and amplitude and wavelength of undulation $A=2 \mu \mathrm{m}$ and $\lambda=30 \mu \mathrm{m}$, respectively. Figure 8 depicts the magnitude of wall shear stress at the wavy wall normalized to the Poiseuille solution for a straight channel with the same width as the effective width of the wavy channel, where effective width is the mean width over one period.

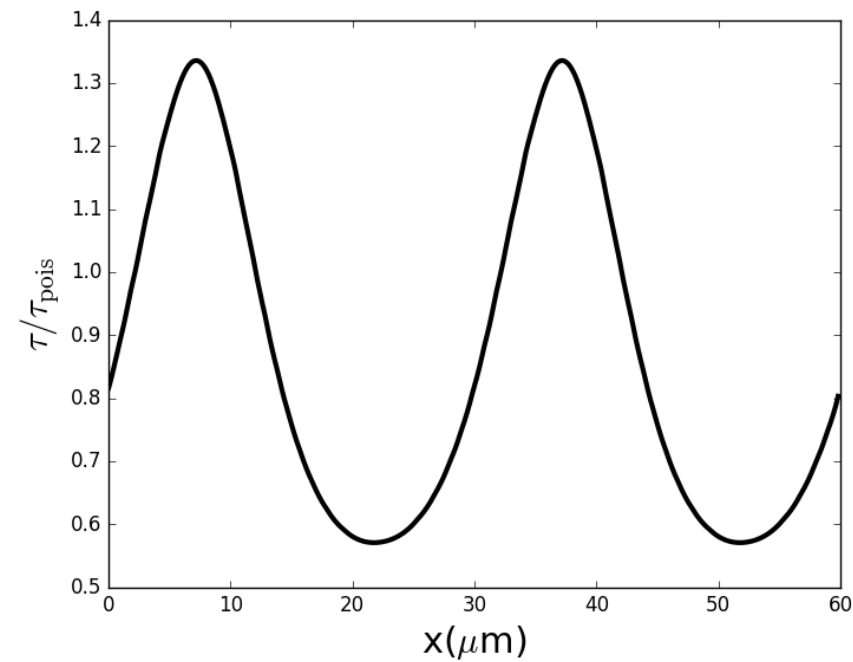

Fig. 8: Normalized wall shear stress along a channel with a boundary given by a sinusoidal function where $\lambda=30 \mu m$ and $A=2 \mu m$.

In agreement with previous studies (Satcher et al. 1992; Barbee et al 1995), the simulations demonstrate that the maximal shear stress can be $> \pm 30 \%$ that of the Poiseuille value for a straight channel with the same effective width, the extremes corresponding to the crests and valleys of the wall. 


\subsection{RBC in a Wavy-Walled Channel}

We examined the effect of confinement for two $C a$ numbers in wavy-walled channels where the waviness of the top wall was in-phase with that of the bottom wall. As the RBC travels across one wavy period, changes in the local flow field result in changes to its morphology. We examined the morphologies obtained and the extremal wall shear stresses. Fig. 9 presents for the lowest and highest reduced areas investigated, the wall shear stress and its extremal values along one period of the wall undulation such that $\tau_{\max }=\max \left(\tau(x) / \tau_{w, \text { nocell }}(x)\right)$ and $\tau_{\min }=\min \left(\tau(x) / \tau_{w, \text { nocell }}(x)\right)$ where $\tau_{w, \text { nocell }}$ is the wall shear stress obtained in the wavy-walled channel in the absence of RBC.

(a)

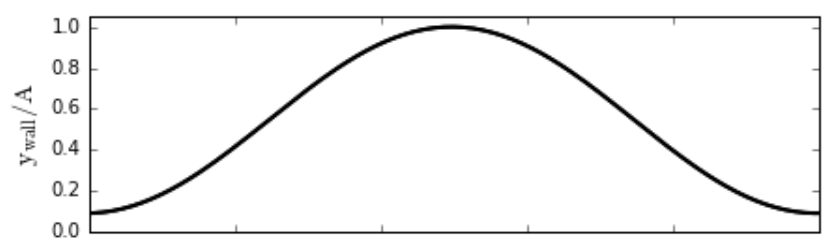

(b)

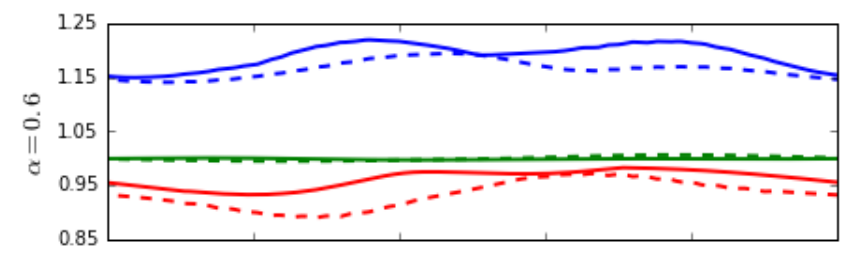

(c)

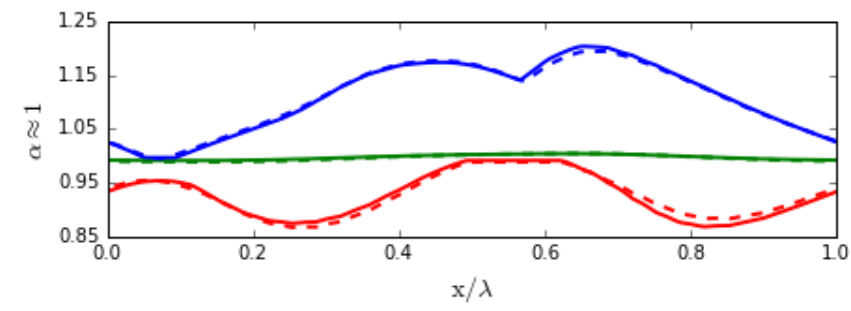

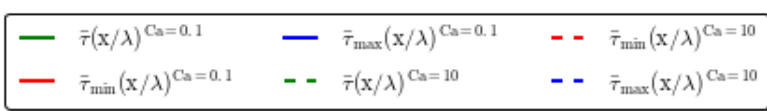

Fig. 9: (a) One period of the wall geometry (b) Average, maximum, and minimum wall shear stress averaged in time a for channel containing a flowing $\mathrm{RBC}$ with either $\alpha=0.6$ (c) or $\alpha \approx 1$ and $C a=0.1$ and $C a=10$. The corresponding morphologies as the RBC transverses the undulating wall are shown in Fig 10a and 11a and Fig $10 \mathrm{e}$.

We see that the average shear stress is roughly equal to the steady-state, continuum solution in the absence of the RBC. However, the time-averaged minimum and maximum shear stresses reveal important differences in subcellular extremal wall shear stress values. In the case of $\alpha \approx 1$, the variation over one period is greatest, the maximum varying greater than $20 \%$ over one period, while in the case of $\alpha=0.6$, there is less than a $10 \%$ difference in extremal values over one period. It has been speculated that subcellular gradients are potentially important in mechanotransduction processes, and our results show that different reduced areas of cells lead to different time-averaged subcellular gradients in extremal wall shear stress values (Satcher et al. 1992; Barbee et al. 1994).

As we did in the case of the flat-walled channel (cf: Figs. 4-6), we examine the behavior of the extremal shear stress as the reduced area is varied. This is performed for the case of a relatively rigid $\mathrm{RBC}(\mathrm{Ca}=0.1)$ and a relatively soft $\mathrm{RBC}(C a=10)$. The extremal wall shear stresses are plotted as a function of reduced area in Fig 10a. 
(a)

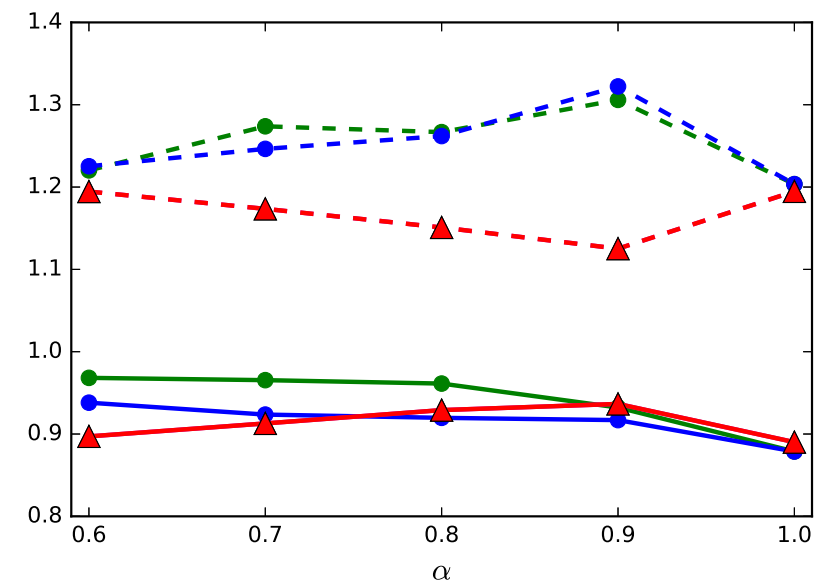

\begin{tabular}{|c|c|c|c|}
\hline $\begin{array}{l}\bullet \tau_{\min , \text { bot }}^{\mathrm{Ca}=0.1} \\
\bullet \begin{array}{l}\mathrm{Ca}=0.1 \\
\mathrm{max}, \mathrm{bot}\end{array}\end{array}$ & $\begin{array}{ll}\bullet & \tau_{\min , \text { top }}^{\mathrm{Ca}=0.1} \\
\bullet & \tau_{\max , \text { top }}^{\mathrm{Ca}=0.1}\end{array}$ & $\begin{array}{l}\Delta \tau_{\min , \text { bot }}^{\mathrm{Ca}=10} \\
\Delta \Delta \tau_{\max , \text { bot }}^{\mathrm{Ca}=0}\end{array}$ & $\begin{array}{l}\Delta \triangle \tau_{\min \text { top }}^{\mathrm{Ca}=10} \\
\Delta \Delta \tau_{\max , \text { top }}^{\mathrm{Ca}=10}\end{array}$ \\
\hline
\end{tabular}

(b)

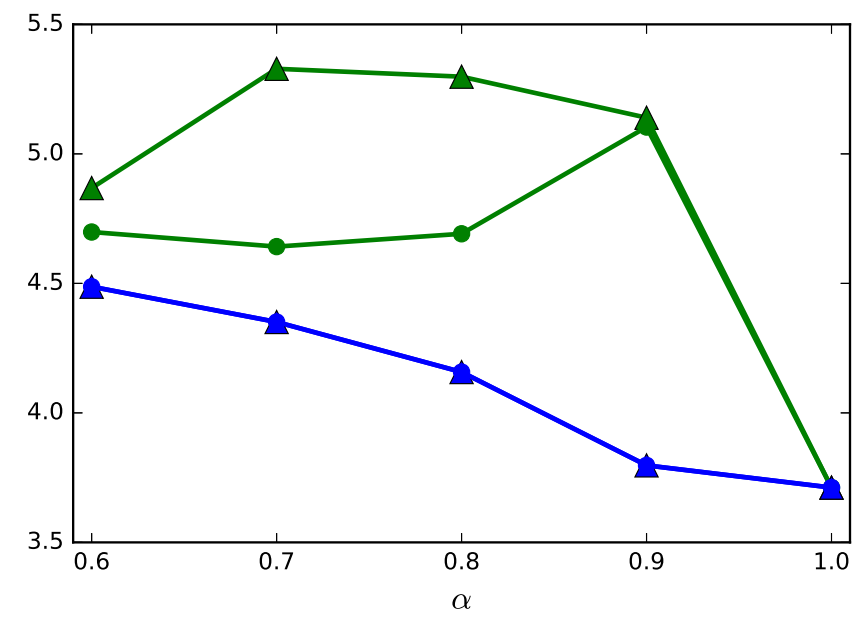

$$
\begin{array}{ll}
\hookrightarrow\left(\mathrm{d}_{\text {min, bot }}^{\mathrm{Ca}=0.1}\right)^{-1} \mathrm{~W}_{\text {eff }} & \leftrightarrow\left(\mathrm{d}_{\text {min, bot }}^{\mathrm{Ca}=10}\right)^{-1} \mathrm{~W}_{\text {eff }} \\
\longrightarrow\left(\mathrm{d}_{\text {min, top }}^{\mathrm{Ca}=0.1}\right)^{-1} \mathrm{~W}_{\text {eff }} & \longrightarrow\left(\mathrm{d}_{\text {min, top }}^{\mathrm{Ca}=10}\right)^{-1} \mathrm{~W}_{\text {eff }}
\end{array}
$$

Fig. 10: (a) Maximum and minimum wall shear stress along the top and bottom walls of a wavy-walled channel as a function of reduced area with $C_{n}=$ $0.42\left(W_{\text {eff }}=12 \mu \mathrm{m}\right)$ and $C a=0.1$ and $C a=10$; (b) The inverse of the normalized minimum distance as a function of reduced area.

Figure 10a shows that the maximum wall shear stress in the wavy wall case is less sensitive to changes in reduced area than in the flat wall case. In extreme, the maximum wall shear stress only varies by $\sim 10 \%$, whereas in the flat wall case it can vary by $\sim 30 \%$ among reduced areas. Waviness reduces the variation in maximum shear stresses observed for different reduced areas; however, the values are in general higher than the equivalent in the straight case. The inverse of the minimum distance normalized to the effective width of the channel is not predictive of the maximum wall shear stress as is suggested by Fig 7 .

(a)

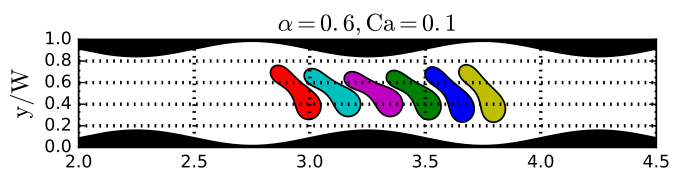

(b)

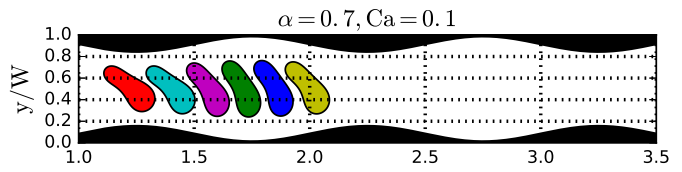

(c)

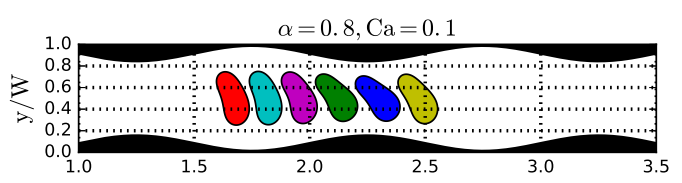

(d)

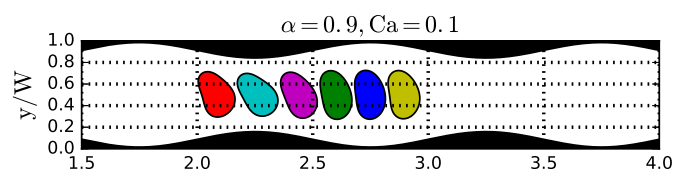

(e)

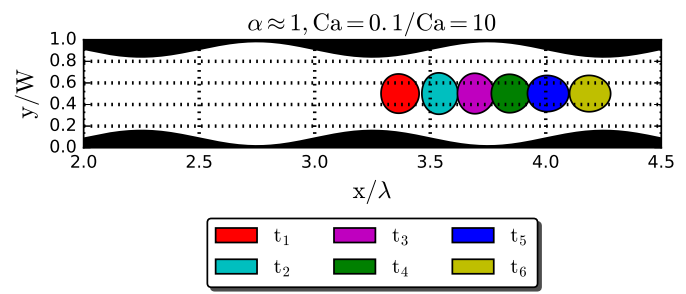

Fig. 11: Six snapshots of the morphological changes of a single RBC as it passes over one wall undulation for different values of reduced area (a) $\alpha=0.6$ (b) $\alpha=$ 0.7 (c) $\alpha=0.8$ (d) $\alpha=0.9$ (e) $\alpha \approx 1$ with $C_{n}=$ $0.42\left(W_{\text {eff }}=12 \mu \mathrm{m}\right)$ and $C a=0.1$

Figure 11 shows snapshots of one RBC as it flows over one period of wall undulation. We see that what would be a stationary morphology in the case of a flat-walled channel is no longer so due to the variation of the flow field along the undulation. Because the RBC is effectively rigid, it does not undergo a noticeable deformation in either the streamwise or perpendicular streamwise directions as it passes through the constriction and expansion of one wall undulation. Instead, we see that the tilt angle of the RBC changes over one period. As the cell approaches the maximal constriction of the two walls, the RBC tilts towards the horizontal axis, and as the cell exits the constriction, the tilt moves toward the vertical axis. 
The results above were for a rigid $\mathrm{RBC}(\mathrm{Ca}=0.1)$. For $\mathrm{Ca}=10$, representing a softer cell, all morphologies obtained are axisymmetric, so the maximum shear stress along the top and bottom walls are identical, as shown in Fig. 12.

(a)

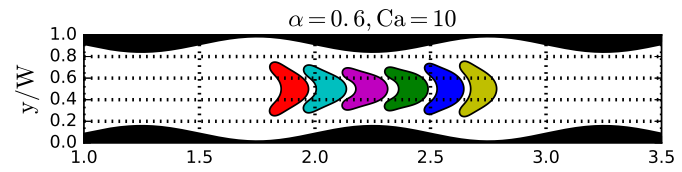

(b)

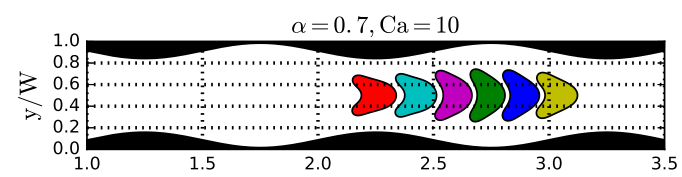

(c)

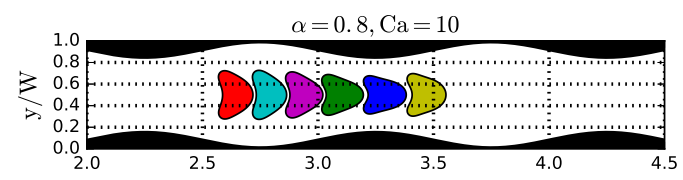

(d)

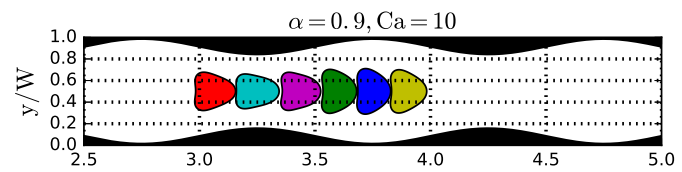

(e)

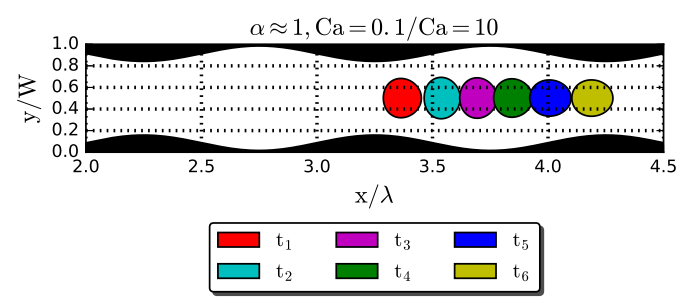

Fig. 12: Snapshots at 6 instants in time showing the morphological changes of a single RBC as it passes over one wall undulation for different values of reduced area (a) $\alpha=0.6$ (b) $\alpha=0.7$ (c) $\alpha=0.8$ (d) $\alpha=0.9$ (e) $\alpha \approx 1$ with $C_{n}=0.42\left(W_{e f f}=12 \mu m\right)$ and $C a=10$.

The morphologies of the RBC as it passes over one wall undulation are always axisymmetric as shown in Fig. 12. We can analyze the passage of the cell over one wall undulation. Before entering the constriction, the cell is stretched. The cell is squashed in the middle of the constriction. Upon exiting, the cell experiences a rebound, whereby the rear-end of the RBC moves faster than the front end so that we see stretching in the perpendicular streamwise direction. The RBC elongates along the streamwise direction in the constriction and expands in the perpendicular streamwise direction in the valley. These same dynamics were also observed for capsules passing through constrictions (Rorai et al. 2015).

All the wavy wall results presented thus far were for the case where the undulations in the top and bottom walls are in phase. In this case, RBCs pass periodically through areas of strong confinement (at peaks) and weak confinement (at valleys). This scenario is unlikely to be the most representative configuration in real situations. This has naturally led us to study the effect of dephasing between the two walls. We dephased the sine curves at the top and the bottom walls of the channel and examined the maximum shear stresses experienced as a function of the dephasing angle such that one of the boundaries was shifted according to the following expression:

$y_{\text {wall }}=\frac{A}{2} \sin \left(\frac{2 \pi x}{\lambda}+2 \pi n\right)+\frac{A}{2}$

where $n$ is a fraction from $[0,1]$. The morphologies of one cell as it passes over one wall undulation are shown in Fig. 13.

(a)

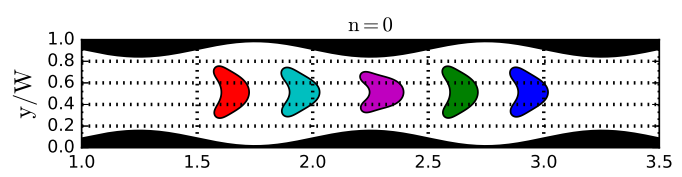

(b)

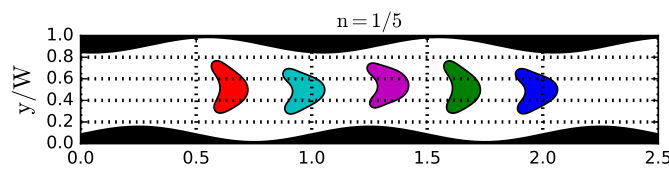

(c)

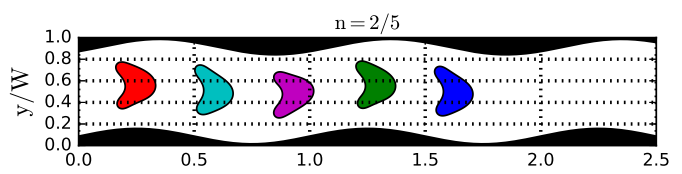

(d)

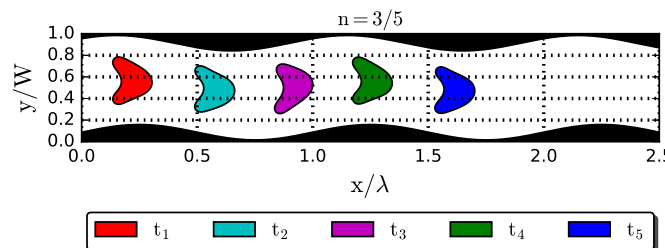

Fig. 13: Five snapshots of the morphology of a single RBC as it passes over one wall undulation for different dephasing angles between the top and bottom walls (a) $n=0$ (b) $n=1 / 5$ (c) $n=2 / 5$ (d) $n=3 / 5$.

We see the effect of dephasing on the morphology of the RBCs in this case where $C a=10$. For this case, when the walls were in-phase, the RBC maintained 
axisymmetric morphology at all times while passing over the length of one period; however, now the RBC shows asymmetric morphologies when the top and bottom walls are out-of-phase. The trailing edge of the parachute elongates in the direction of the further wall. In order to see what effect the dephasing has on the wall shear stress, we plot the maximum wall shear stress as a function of the dephasing angle in Fig. 14.

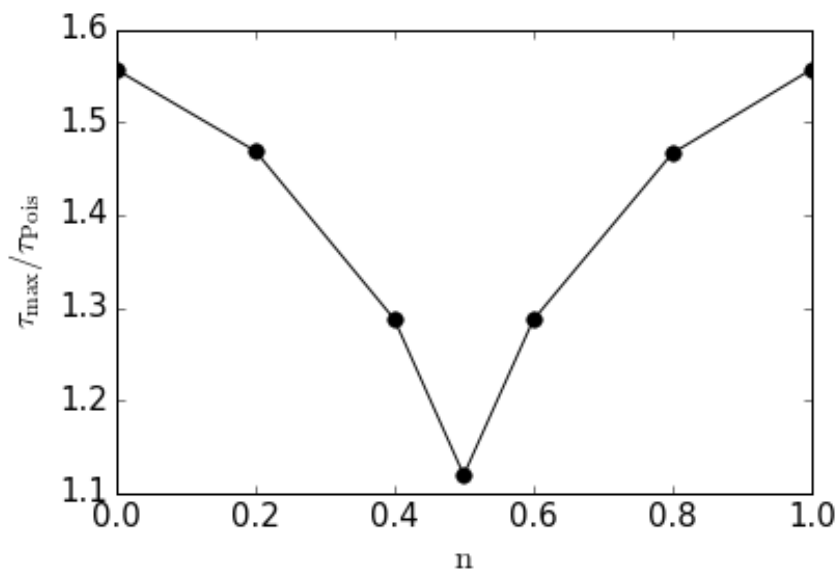

Fig. 14: Maximum wall shear stress normalized to the Poiseuille solution (for a flat-walled channel having the same effective width) along the channel walls as a function of the degree of dephasing, $n$.

The maximum wall shear stress takes its largest value when the top and bottom walls are in-phase and its smallest value when the walls are half a wavelength out-of-phase. We observe that the effect of dephasing can decrease the maximum wall shear stress in the channel by nearly $30 \%$. Although the properties of the RBCs are held constant as is the applied pressure gradient and the effective width of the channel, the local changes to the flow field resulting from undulation dephasing have a significant effect on the observed wall shear stress. Thus, even small changes to the geometry of the endothelial surface have important ramifications on RBC morphology and the resulting wall shear stress.

\section{Discussion}

We have studied the effect of morphological changes in RBCs, confinement, capillary number, reduced area, and vessel wall geometry on the wall shear stresses in microvessels. We have computed the possible shear stress fluctuations that each of these physical parameters might induce using a simulation model for flow in the microvasculature with the idea that these fluctuations could be important in mechanotransduction events. Our results have demonstrated that the presence of RBCs has a significant effect on the wall shear stress. Not surprisingly, the effect of RBCs on wall shear stress becomes more pronounced with increased confinement (i.e. smaller vessels), and the effect becomes more dynamic under conditions that induce fluctuations in RBC morphology. Interestingly, we have determined that the maximum wall shear stress is correlated to the minimum distance between the cell and the adjacent wall in small confinements. This observation runs counter to previous notions that the wall shear stress is directly proportional to the local distance between the RBC and the wall (i.e. $\tau=\mu V_{R B C} / \delta$ where $\delta$ is the local distance between the RBC and the wall) (Namgung et al. 2011).

Another important result of the present work stems from accounting for the waviness of the endothelial surface. We determined that in the case of wavy walls, the exact geometry of the endothelium changes RBC dynamics and hence has a significant impact on the shear stresses experienced along the wall, highlighting the importance of experimental studies focusing on the topography of confluent endothelial monolayers (Chtcheglova et al. 2010) so that a more realistic geometry could be incorporated into future modeling. We have also seen that in the cases studied, the effect of waviness is to reduce the variation in maximal wall shear stress among different reduced areas when compared to the flat wall counterpart.

Our results demonstrating the significance of interactions between RBCs and endothelial cell topography in determining the wall shear stress on the endothelial surface have significant implications for studies of flowmediated endothelial mechanobiology. The vast majority of studies of endothelial responses to flow do not account for the impact of RBCs. In light of the present findings of the highly dynamic nature of RBC morphology as they flow within microvessels, one would expect the incorporation of RBCs would lead to a local shear stress environment that constantly changes in time. The implications of such rapid dynamics on endothelial cell structure and function certainly merit investigation.

A limitation of the present work is that it has focused on a single RBC within a microvessel. Naturally, future investigations need to account for multiple RBCs flowing in a disordered manner and the effect of interactions among RBCs on the wall shear stress within the vessel. 3D simulations would lead us to a map of wall shear stress for the entire vessel wall, although these 2D simulations do give us a qualitative framework to approach more complex, realistic models. We note, however, that in smaller microvessels, RBCs often flow in a single file, in which case the present results become more directly physiologically relevant. However, we believe that these results provide useful qualitative information to be applied to more complex and realistic 
cases of many RBCs flowing in a disordered manner in channel flow.

Acknowledgements Brenna E. Hogan is supported by a doctoral fellowship from Ecole Polytechnique. This research is funded in part by a permanent endowment in Cardiovascular Cellular Engineering from the AXA Research Fund, the Centre National d'Etudes Spatiales (CNES), and the FrenchGerman university programme (Living Fluids, Grant CFDAQ1-14).

\section{References}

Aarts, Piet A.M.M., Robert M. Heethaar, and Jan J. Sixma (1984). "Red Blood Cell Deformability Influences Platelets-Vessel Wall Interaction in Flowing Blood". Blood 64.6, pp. 1228-1233.

Bagchi, Prosenjit (2007). "Mesoscale simulation of blood flow in small vessels". Biophysical Journal 92.6, pp. 1858 1877.

Barabino, Gilda A, Larry V McIntire, Suzanne G Eskin, David A Sears, and Mark Udden (1987). "Endothelial Cell Interactions with Sickle Cell, Sickle Trait, Mechanically Injured, and Normal Erythrocytes Under Controlled Flow". Blood 70.1, pp. 152157.

Barakat, Abdul I. (1999). "Responsiveness of vascular endothelium to shear stress: Potential role of ion channels and cellular cytoskeleton (review)". International journal of molecular medicine 4, pp. 323332.

Barbee, K A, T Mundel, R Lal, and P F Davies (1995). "Subcellular distribution of shear stress at the surface of flow-aligned and nonaligned endothelial monolayers". American Journal of Physiology-Heart and Circulatory Physiology 268.4, H1765-H1772.

Barbee, Kenneth A, Peter F Davies, and Ratneshwar. Lal (1994). "Shear Stress-Induced Reorganization of the Surface Topography of Living Endothelial Cells Imaged by Atomic Force Microscopy". Cardiovascular Research 74, pp. 163-171.

Baskurt, Ok (2012). "Red Blood Cell Mechanical Stability". 2012 World Congress on Engineering and Technology 2012.October, pp. 8-10.

Bouzidi, M'hamed, Mouaouia Firdaouss, and Pierre Lallemand (2001). "Momentum transfer of a Boltzmannlattice fluid with boundaries". Physics of Fluids 13.11, pp. 3452-3459.

Chtcheglova, Lilia A, Linda Wildling, Jens Waschke, Detlev Drenckhahn, and Peter Hinterdorfer Afm (2010). "AFM Functional Imaging on Vascular Endothelial Cells". Journal of Molecular Recognition 23.6, p. 589 .
Culver, James C. and Mary E. Dickinson (2010). "The effects of hemodynamic force on embryonic development". Microcirculation 17.3, pp. 164-178.

Davies, Peter F. (1995). "Flow-Mediated Endothelial Mechanotransduction". Physiol Rev 75.3, pp. 519560 .

Diez-Silva, Monica, Ming Dao, Jongyoon Han, ChweeTeck Lim, and Subra Suresh (2010). "Shape and Biomechanical Characteristics of Human Red Blood Cells in Health and Disease." MRS bulletin / Materials Research Society 35.5, pp. 382-388.

Dondorp, Arjen M., Emsri Pongponratn, and Nicholas J. White (2004). "Reduced microcirculatory flow in severe falciparum malaria: Pathophysiology and electron-microscopic pathology". Acta Tropica 89.3, pp. 309-317.

Fedosov, D. A., B. Caswell, S. Suresh, and G. E. Karniadakis (2011a). "Quantifying the biophysical characteristics of Plasmodium-falciparum-parasitized red blood cells in microcirculation". Proceedings of the National Academy of Sciences 108.1, pp. 35-39.

Fedosov, Dmitry A., Bruce Caswell, and George Em Karniadakis (2011b). "Wall shear stress-based model for adhesive dynamics of red blood cells in malaria". Biophysical Journal 100.9, pp. 2084-2093.

Freund, Jonathan B. and Julien Vermot (2014). "The wall-stress footprint of blood cells flowing in microvessels". Biophysical Journal 106.3, pp. 752-762.

García-Cardeña, G and BR Slegtenhorst (2016). "Hemodynamic Control of Endothelial Cell Fates in Development". Annual Review of Cell and Developmental Biology 6.32, pp. 633-648.

Ghigliotti, Giovanni, Hassib Selmi, Lassaad El Asmi, and Chaouqi Misbah (2012). "Why and how does collective red blood cells motion occur in the blood microcirculation?" Physics of Fluids 24.10.

Hahn, C and M A Schwartz (2009). "Mechanotransduction in vascular physiology and atherogenesis". Nat Rev Mol Cell Biol 10.1, pp. 53-62.

Kaoui, Badr, Jens Harting, and Chaouqi Misbah (2011). "Two-dimensional vesicle dynamics under shear flow: Effect of confinement". Physical Review E - Statistical, Nonlinear, and Soft Matter Physics 83.6.

Kroll, Michael H., J. David Hellums, Larry V. McIntire, Andrew I. Schafer, and Joel L. Moake (1996). "Platelets and Shear Stress". Blood 88.5, pp. 15251542.

Krüger, Timm, Fathollah Varnik, and Dierk Raabe (2009). "Shear stress in lattice Boltzmann simulations". Physical Review E 79.4, pp. 1-15.

Namgung, Bumseok, Peng Kai Ong, Paul C. Johnson, and Sangho Kim (2011). "Effect of cell-free layer 
variation on arteriolar wall shear stress". Annals of Biomedical Engineering 39.1, pp. 359-366.

Oberleithner, Hans, Thomas Ludwig, Christoph Riethmüller, Uta Hillebrand, Lars Albermann, Claudia Schäfer, Victor Shahin, and Hermann Schillers (2004). "Human Endothelium: Target for Aldosterone". Hypertension 43.5, pp. 952-956.

Oulaid, Othmane and Junfeng Zhang (2015). "Temporal and Spatial Variations of Wall Shear Stress in the Entrance Region of Microvessels". Journal of Biomechanical Engineering 137.6, p. 061008.

Peskin, Charles S. (2002). "The immersed boundary method". Acta Numerica 11, pp. 479-517.

Pontrelli, Giuseppe, Carola S. König, Ian Halliday, Timothy J. Spencer, Michael W. Collins, Quan Long, and Sauro Succi (2011). "Modelling wall shear stress in small arteries using the Lattice Boltzmann method: Influence of the endothelial wall profile". Medical Engineering and Physics 33.7, pp. 832-839.

Roman, Beth L and Kerem Pekkan (2015). "Mechanotransduction in Embryonic Vascular Development". 11.8, pp. 1149-1168.

Rorai, Cecilia, Antoine Touchard, Lailai Zhu, and Luca Brandt (2015). "Motion of an elastic capsule in a constricted microchannel". The European physical journal. E, Soft matter 38.5, p. 134.

Satcher, R. L., S. R. Bussolari, M.A. Gimbrone Jr., and C. F. Dewey Jr. (1992). "The Distribution of Fluid Forces on Model Arterial Endothelium Using Computational Fluid Dynamics". J Biomech Eng 114.309-316.

Shen, Zaiyi, Alexander Farutin, Marine Thiébaud, and Chaouqi Misbah (2017). "Interaction and rheology of vesicle suspensions in confined shear flow". Phys. Rev. Fluids 2.10, p. 103101.

Succi, Sauro (2001). The Lattice Boltzmann Equation for Fluid Dynamics and Beyond. Oxford University Press.

Tahiri, N., T. Biben, H. Ez-Zahraouy, A. Benyoussef, and C. Misbah (2013). "On the problem of slipper shapes of red blood cells in the microvasculature". Microvascular Research 85, pp. 40-45.

Tsubota, Ken Ichi and Shigeo Wada (2010). "Effect of the natural state of an elastic cellular membrane on tank-treading and tumbling motions of a single red blood cell". Physical Review E - Statistical, Nonlinear, and Soft Matter Physics 81.1.

Tsubota, Ken-ichi, Shigeo Wada, and Takami Yamaguchi (2006). "Particle method for computer simulation of red blood cell motion in blood flow". Computer Methods and Programs in Biomedicine 83.2, pp. 139 146.
Uzarski, Joseph S, Edward W Scott, and Peter S Mcfetridge (2013). "Adaptation of Endothelial Cells to Physiologically-

, Modeled, Variable Shear Stress". PLoS One 8.2.

Vlahovska, Petia M., Thomas Podgorski, and Chaouqi Misbah (2009). "Vesicles and red blood cells in flow: From individual dynamics to rheology". Comptes Rendus Physique 10.8, pp. 775-789.

Vlahovska, Petia M., Dominique Barthes-Biesel, and Chaouqi Misbah (2013). "Flow dynamics of red blood cells and their biomimetic counterparts". Comptes Rendus Physique 14.6, pp. 451-458.

Wu, Tenghu and James J. Feng (2013). "Simulation of malaria-infected red blood cells in microfluidic channels: Passage and blockage". Biomicrofluidics 7.4 , pp. $1-18$.

Xiong, Wenjuan and Junfeng Zhang (2010). "Shear stress variation induced by red blood cell motion in microvessel". Annals of Biomedical Engineering 38.8, pp. 2649-2659.

Xu, Zhensong, Yi Zheng, Xian Wang, Nadine Shehata, Chen Wang, and Yu Sun (2018). "Stiffness increase of red blood cells during storage". Microsystems $\mathscr{E}$ Nanoengineering 4.March 2017, p. 17103.

Ye, Ting, Nhan Phan-Thien, Boo Khoo, and C T Lim (2014). "Numerical modelling of a healthy/malariainfected erythrocyte in shear flow using dissipative particle dynamics method". Journal of Applied Physics 115, p. 224701.

Ye, Ting, Nhan Phan-Thien, and Chwee Teck Lim (2015). "Particle-based simulations of red blood cellsA review". Journal of Biomechanics 49.11, pp. 22552266.

Yin, Xuewen and Junfeng Zhang (2012). "Cell-free layer and wall shear stress variation in microvessels". Biorheology 49.4, pp. 261-270.

Zhang, Junfeng, Paul C. Johnson, and Aleksander S. Popel (2007). "An immersed boundary lattice Boltzmann approach to simulate deformable liquid capsules and its application to microscopic blood flows". Physical Biology 4.4, pp. 285-295. 